Pacific Journal of Mathematic 


\title{
SEMIGROUPS, HORN SENTENCES AND ISOLIC STRUCTURES
}

\author{
ERIK ELLENTUCK
}

\begin{abstract}
We introduce a special type of semigroup whose properties are abstracted from the theory of cardinal numbers under addition. A very general theorem about the behavior of universal Horn sentences in such semigroups is obtained and then applied to isols, recursive equivalence types, cardinal numbers, and Dedekind cardinals, the last two being in set theory without the axiom of choice.
\end{abstract}

1. Introduction. In this paper we introduce a special kind of semigroup which is related to certain cardinal like structures under a single binary operation. We call them Tarski semigroups (TSGs) and discuss their general properties in $\S 2$. There we show that a canonical partial order can be induced on a TSG and that every TSG can be canonically embedded in a torsion free Abelian group (TFAG). In $\S 3$ we give necessary and sufficient conditions that every universal Horn sentence which holds in the nonnegative integers under addition also holds in a TSG. We also do the same for the positive integers under multiplication. Our proofs rely heavily on vector space methods. Section 4 applies these results to definite semigroups constructed from cardinal numbers and recursive equivalence types. As a specific example consider the isols $\Lambda$. We show that although $(\Lambda,+, 0)$ and $(\Lambda-\{0\}, \cdot, 1)$ share the same universal Horn sentences, there is an $\mathrm{AE}$ Horn sentence which distinguishes them. Uniformity is again obtained by showing that their canonical TFAGs have the same first order theory which is identical to that of the positive rationals under multiplication. The same results are obtained for the Dedekind cardinals. We find these results surprising on two counts. First, we have a structure built from the integers, whose first order theory is the same as that of a corresponding structure built from the isols. Usually it is only the universal Horn sentences which remain unchanged. Second, we have a structure built from the Dedekind cardinals whose first order theory is complete. In most other constructions incompleteness is the rule. Section 4 concludes with the result of $A$. Nerode (subsequently obtained by the author), that $n A \leqq n B \rightarrow A \leqq B$ where $n$ is a positive integer and $A, B$ are recursive equivalence types. This result is not isolated, but fits into our general semigroup analysis.

2. Semigroups. Let $\mathscr{S}=(S,+, 0)$ be a groupoid, that is $S$ is a 
nonempty set, + is a binary operation on $S$, and 0 is a distinguished element of $S$. If $\omega=$ the nonnegative integers, $n \in \omega$, and $x \in S$ we define a scalar product $n x$ by the recursion $0 x=0$ and $(n+1) x=$ $(n x)+x . \mathscr{S}$ is called a Tarski semigroup $(T S G)$ if it satisfies the universal closures of (1) thru (6) below.

$$
\begin{gathered}
x+(y+z)=(x+y)+z . \\
x+0=x=0+x . \\
x+y=y+x . \\
x+y=0 \longrightarrow x=0 . \\
x+z=y+z \longrightarrow x=y . \\
n x=n y \longrightarrow x=y \text { for } 0<n<\omega .
\end{gathered}
$$

Such structures are meant to reflect some of the algebraic properties of cardinal numbers and recursive equivalence types. Let $\Gamma=$ the cardinals, $\Delta=$ the Dedekind cardinals, $\Omega=$ the recursive equivalence types, and $\Lambda=$ the isols. If we consider each of these structures with its canonical + and 0 , then all satisfy (1) thru (4), (5) is the defining condition for both $\Delta$ and $A$, Dekker-Myhill have show that (6) holds in $\Lambda$ (cf. [2]), Friedberg has shown that (6) holds in $\Omega$ (cf. [6]), and Bernstein, Sierpinski, and Tarski have all shown that (6) holds in $\Gamma$ (a fortiori in $\Delta$ as a substructure of $\Gamma$ ) (cf. [1], [11], and [14]).

In $\mathscr{S}$ we define the canonical inequality $x \leqq y \equiv_{D F}(\exists z) y=x+z$. Then every TSG satisfies the universal closures of (7.1) thru (7.11) below.

$$
\begin{gathered}
0 \leqq x \\
x \leqq x . \\
x \leqq y \wedge y \leqq x \longrightarrow x=y . \\
x \leqq y \wedge y \leqq z \longrightarrow x \leqq z . \\
x \leqq y \longrightarrow x+z \leqq y+z . \\
x+z \leqq y+z \longrightarrow x \leqq y . \\
(m+n) x=(m x)+(n x) . \\
n(x+y)=(n x)+(n y) . \\
m(n x)=(m n) x . \\
m \leqq n \longrightarrow m x \leqq n x .
\end{gathered}
$$




$$
x \leqq y \longrightarrow n x \leqq n y .
$$

(7.1) thru (7.11) with the exceptions of (7.3) and (7.6) can be proved using only (1) thru (3), and (7.6) uses (5) in an obvious way. To prove (7.3) note that if $x \leqq y \wedge y \leqq x$ then there exist $u, v$ such that $x+u=y$ and $y+v=x$ so that $x+(u+v)=(x+u)+v=$ $y+v=x=x+0$ by (1) and (2). Then $u+v=0$ by (5), $u=0$ by (4), and $x=y$ by (2). This reflects the fact that (7.3), a CantorBernstein like theorem, is easy to prove for $\Delta$ and $\Lambda$, but considerably harder to prove for $\Gamma$ and $\Omega$ (which do not satisfy (5)). Let us consider

$$
n x \leqq n y \longrightarrow x \leqq y \text { for } 0<n<\omega
$$

and ask whether (8) follows from (6) in the same trivial way that (7.6) follows from (5). The answer is no, and in section 4 we give some important examples of TSGs which do not satisfy (8). Thus (8) was not added to our definition of a TSG in order to include these examples in our study.

Next we embed our TSG $\mathscr{S}$ into a torsion free Abelian group (TFAG) $\mathscr{S}^{*}$. In $\mathscr{S}$ define $(x, y) \sim(u, v) \equiv_{D F} x+v=y+u$. Thus $\mathscr{S}$ will satisfy the universal closures of (9.1) thru (9.11) below.

$$
\begin{gathered}
(x, y) \sim(x, y) . \\
(x, y) \sim(u, v) \longrightarrow(u, v) \sim(x, y) . \\
(x, y) \sim(u, v) \wedge(u, v) \sim(a, b) \longrightarrow(x, y) \sim(a, b) .
\end{gathered}
$$

An algebraic structure is imposed on these pairs by defining $(x, y)+$ $(u, v)=_{D F}(x+u, y+v)$. Then

$$
\begin{gathered}
((x, y)+(u, v))+(a, b)=(x, y)+((u, v)+(a, b)) \\
(x, y)+(u, v)=(u, v)+(x, y) . \\
(x, y) \sim\left(x^{\prime}, y^{\prime}\right) \longrightarrow(x, y)+(u, v) \sim\left(x^{\prime}, y^{\prime}\right)+(u, v) . \\
(x, x) \sim(0,0) . \\
(x, y)+(0,0)=(x, y) . \\
(x, y)+(y, x) \sim(0,0) .
\end{gathered}
$$

We define a scalar product $n(x, y)$ on pairs in exactly the same way that we defined a similar product in $\mathscr{S}$. Then

$$
n(x, y)=(n x, n y) \text {. }
$$

$$
n(x, y) \sim(0,0) \longrightarrow(x, y) \sim(0,0) \text { for } 0<n<\omega .
$$


(9.1) thru (9.11) with the exceptions of (9.3) and (9.11) can be proved using only (1) thru (3), and (9.11) uses (6) in an obvious way. It is interesting to note that (7.3) and (9.3) both of which appear early in their respective constructions, seem to require (5).

Now define $[x, y]={ }_{D F}\{(u, v) \mid(u, v) \sim(x, y)\}, S^{*}={ }_{D F}\{[x, y] x, y \in S\}$, $[x, y]+*[u, v]=_{D F}[x+u, y+v], 0^{*}={ }_{D F}[0,0]$, and $\mathscr{S}^{*}={ }_{D_{F}}\left(S^{*},+^{*}, 0^{*}\right)$. Using (9.1) thru (9.11) we can show that $+^{*}$ and $0^{*}$ are well defined and that $\mathscr{S}^{*}$ is a TFAG. Next we define a function $h: S \rightarrow S^{*}$ by $h(x)={ }_{D F}[x, 0]$ and note that

$$
\begin{gathered}
{[x, 0]=[y, 0] \longrightarrow x=y,} \\
x+y=z \equiv[x, 0]+{ }^{*}[y, 0]=[z, 0],
\end{gathered}
$$

which together imply that $h$ is an embedding of $\mathscr{S}$ into $\mathscr{S}^{*}$. Thus, w.l.g., we shall assume that $S \subseteq S^{*}$, the restriction of $+^{*}$ to $S$ is + , and that $0=0 *$.

Although the material of this section is quite routine, we have run thru it again in order to emphasize the importance of the Dedekind property (5).

3. Horn sentences. We introduce a first order language $L$ in order to discuss the elementary properties of TSGs and TFAGs. The primitive symbols of $L$ consist of an infinite list of individual variables $v_{0}, v_{1}, \cdots$, a binary predicate $=$ denoting equality, a binary functor + denoting addition, and an individual constant 0 denoting the identity element. Terms, formulas, sentences, and so forth are taken from current usage. A system of equations is the conjunction of some finite number of atomic formulas of $L$. A universal Horn sentence is the universal closure of a formula of the form $s \rightarrow s^{\prime}$ where $s$ and $s^{\prime}$ are systems of equations. Strictly speaking, a universal Horn sentence should have a conjunction of implications of the form $s \rightarrow s^{\prime}$ for its matrix, however since our prefix is universal we lose no generality by requiring that our matrix consist of a single conjunct.

We first consider the question of what universal Horn sentences are satisfied in a given TSG $\mathscr{S}=(S,+, 0)$. Let $\omega^{+}$be the TSG $(\omega,+, 0) . \mathscr{S}$ is said to satisfy the Tarski condition ${ }^{+}\left(T C^{+}\right)$if every system of equations in the variables $v_{0}, \cdots, v_{k}$ having a solution $x_{0}, \cdots, x_{k}$ in $\mathscr{S}$ with $x_{0} \neq 0$ also has a solution $x_{0}^{\prime}, \cdots, x_{k}^{\prime}$ in $\omega^{+}$with $x_{0}^{\prime} \neq 0$. $\mathscr{S}$ is said to have the Horn transfer property ${ }^{+}\left(H T P^{+}\right)$if every universal Horn sentence which is true in $\omega^{+}$is also true in $\mathscr{S}$. Our first important result is

THEOREM 1. A TSG $\mathscr{S}$ has the $H T P^{+}$if and only if it satisfies the $T C^{+}$. 
Proof. In one direction our theorem is quite easy. Assume that $\mathscr{S}$ has the $\mathrm{HTP}^{+}$and that $s$ is a system of equations in the variables $v_{0}, \cdots, v_{k}$. If $s$ has no solution $x_{0}^{\prime}, \cdots, x_{k}^{\prime}$ in $\omega^{+}$with $x_{0}^{\prime} \neq 0$ then

$$
\left(\forall v_{0}, \cdots, v_{k}\right)\left(s \longrightarrow v_{0}=0\right)
$$

is true in $\omega^{+}$and hence by $\mathrm{HTP}^{+}$also true in $\mathscr{S}$. But then $s$ can have no solution $x_{0}, \cdots, x_{k}$ in $\mathscr{S}$ with $x_{0} \neq 0$. For the converse assume that $\mathscr{S}$ satisfies the $\mathrm{TC}^{+}$, that $s$ and $s^{\prime}$ are systems of equations in the variables $v_{0}, \cdots, v_{k}$ and that $\mathfrak{A}$ is a universal Horn sentence which is true in $\omega^{+}$and has the form

$$
\left(\forall v_{0}, \cdots, v_{k}\right)\left(s \longrightarrow s^{\prime}\right) \text {. }
$$

The following terminology is convenient. A formula is TSG-valid if its universal closure is true in every TSG. Two formulas are TSGequivalent if their biconditional is TSG-valid. Thus for every term $t$ which appears in $\mathfrak{A}$ we can find a term $t^{\prime}$ of the form $\sum_{i \leqq k} n_{i} v_{i}$, where each $n_{i} \in \omega$, such that $t=t^{\prime}$ is TSG-valid.

We must show that $\mathfrak{A}$ is true in $\mathscr{S}$. This is done by an induction on the number of variables $k+1$ which appear in $\mathfrak{A}$. For $k=0$ every equation is TSG-equivalent to one of the form $m v_{0}=n v_{0}$. If $m=n$ this is TSG-equivalent to $0=0$, and if $m \neq n$, say $m>n$, it is TSGequivalent to $(m-n) v_{0}=0$ by (5), and to $v_{0}=0$ by either (4) or (6). Since $\mathfrak{A}$ is true in $\omega^{+}$the only case ruled out is where $s$ is replaced by $0=0$ and $s^{\prime}$ by $v_{0}=0$. But then $\mathfrak{A}$ is clearly true in $\mathscr{S}$.

Before going on to the inductive step let us put our problem into vector space notation. Consider a TSG $\mathscr{S}$ as embedded into $\mathscr{S}^{*}$. Since subtraction is possible in $\mathscr{S}^{*}$, for every equation $e$ which appears in $\mathfrak{A}$ we can find a term $t$ of the form $\sum_{i \leq k} n_{i} v_{i}$, where each $n_{i} \in\left(\omega^{+}\right)^{*}$, such that $e$ is true in $\mathscr{S}$ if and only if $t=0$ is true in $\mathscr{S}^{*}$ when the $v_{i}$ are assigned values in $S$. Note that we have identified the group $\left(\omega^{+}\right)^{*}$ with its underlying domain, and shall do so in other situations when it is inconvenient to introduce further notation. Conversely every such equation $t=0$ can be given a meaning in $\mathscr{S}$ by separating $t$ into its positive and negative parts and then transposing. Thus for every system of equations $s$ we can find a matrix $A$, whose entries belongs to $\left(\omega^{+}\right)^{*}$, such that $s$ is TSGequivalent to $A v=0$. In $A v=0$ we interpret $v=\left(v_{0}, \cdots, v_{k}\right)$ as a vector, $A v$ as ordinary matrix multiplication, and 0 as the zero vector, where possibly some of the computation is going on in $\mathscr{S}^{*}$. Combining these results will give us matrices $A$ and $B$ such that for any TSG $\mathscr{S}$, the truth of (12) in $\mathscr{S}$ is equivalent to the truth in $\mathscr{S}$ of

$$
(\forall v)(A v=0 \longrightarrow B v=0) .
$$


W.l.g. assume (12) is already in the form given by (13).

Now assume $k>0$ and that we have established the $\mathrm{HTP}^{+}$for sentences with fewer than $k+1$ variables. Two cases arise.

Case 1. There is a vector $y$, all components of which belong to $w-\{0\}$, such that $A y=0$. We claim that $\mathfrak{X}$ is true in $\left(\omega^{+}\right)^{*}$. Let $x$ be any $k+1$-tuple of rational integers such that $A x=0$. Choose positive $n$ such that $x+n y$ is positive in all components. Then $A(x+n y)=(A x)+n(A y)=0$ and hence since (13) is true in $\omega^{+}$, both $B(x+n y)=0$ and $B y=0$. But $B(x+n y)=(B x)+n(B y)=B x=0$. Thus $\mathfrak{A}$ holds in $\left(\omega^{+}\right)^{*}$. Next we claim that $\mathfrak{A}$ is true in the field of rational numbers $\mathscr{R}=(Q,+, \cdot, 0,1)$. Consider a vector $x=\left(x_{0}, \cdots\right.$, $x_{k}$ ) where each $x_{i}$ has the form $y_{i} / z_{i}$ with $y_{i} \in\left(\omega^{+}\right)^{*}$ and $z_{i} \in \omega-\{0\}$. W.l.g. we may assume that the $z_{i}$ have a common positive value $d$. Let $y=\left(y_{0}, \cdots, y_{k}\right)$ and assume that $A x=0$. Then $d^{-1}(A y)=A\left(d^{-1} y\right)=$ $A x=0$ and hence $A y=0$. Since (13) is true in $\left(\omega^{+}\right)^{*}$ we have $B y=0$ and hence $B x=B\left(d^{-1} y\right)=d^{-1}(B y)=0$. Thus $\mathfrak{A}$ is true in $\mathscr{R}$.

We can interpret the truth of (13) in $\mathscr{R}$ in the following geometrical terms. In the vector space $\mathscr{V}$ consisting of all $k+1$-tuples of rational numbers every vector which is perpendicular to each row of $A$ is also perpendicular to each row of $B$. We claim that $R_{B}$, the subspace of $\mathscr{V}$ spanned by the rows of $B$, is contained in $R_{A}$, the subspace of $\mathscr{V}$ spanned by the rows of $A$. For any subspace $\mathscr{C}$ of $\mathscr{V}$ let $\mathscr{U}^{\perp}$ be the orthogonal complement of $\mathscr{C}$ in $\mathscr{T}$. Then by hypothesis $\left(R_{A}\right)^{\perp} \cong\left(R_{B}\right)^{\perp}$ so that $\left(R_{B}\right)^{\perp \perp} \subseteq\left(R_{A}\right)^{\perp \perp}$. But $\mathscr{U}^{\perp \perp}=\mathscr{\mathscr { C }}$ which proves our claim. Denote the rows of $A$ by $a_{j}, j<n$, and the rows of $B$ by $b_{i}, i<m$. Then there exist rational numbers $r_{i j}$ such that $b_{i}=\sum_{j<n} r_{i j} a_{j}$. By removing a positive common denominator $d$ if necessary, we can find a matrix $D$, the entries of which are rational integers, such that

$$
d B=D A,
$$

the product on the left (right) being respectively scalar (matrix) multiplication.

We now show that $\mathfrak{A}$ is true in $\mathscr{S}$. Let $x$ be a vector with components from $S$ such that $A x=0$. Then $d(B x)=D(A x)=0$ by (14). In general $B x \in S^{*}$, but since $\mathscr{S}^{*}$ is torsion free we get $B x=0$. Thus $\mathfrak{A}$ is true in $\mathscr{S}$. Notice that up to now we have used neither our inductive hypothesis nor the $\mathrm{TC}^{+}$. However they will be used in

Case 2. There is no vector $y$, all entries of which belong to $\omega-\{0\}$, such that $A y=0$. Suppose that for each $i \leqq k$ there is a vector $y_{i}$ such that each component of $y_{i}$ belongs to $\omega$, the $i$-th one 
being positive, and $A y_{i}=0$. Then $y=\sum_{i \leq k} y_{i}$ is positive in all components and $A y=0$, contradicting the fact that we are in Case 2. Thus for some $j \leqq k(\forall v)\left(A v=0 \rightarrow v_{j}=0\right)$ is true in $\omega^{+}$. By renaming variables we may assume that $j=0$ so that by $\mathrm{TC}^{+}$

$$
(\forall v)\left(A v=0 \longrightarrow v_{0}=0\right)
$$

is also true in $\mathscr{S}$. Let $A^{\prime}$ and $B^{\prime}$ be the matrices obtained from $A$ and $B$ respectively by deleting their first columns. Then it is easy to see that the truth of (13) and (15) in $\omega^{+}$implies the truth of

$$
\left(\forall v^{\prime}\right)\left(A^{\prime} v^{\prime}=0 \longrightarrow B^{\prime} v^{\prime}=0\right)
$$

in $\omega^{+}$where $v^{\prime}=\left(v_{1}, \cdots, v_{k}\right)$. Now let $x=\left(x_{0}, \cdots, x_{k}\right)$ be a vector with components from $S$ such that $A x=0$. Then $x_{0}=0$ by (15) and hence $A^{\prime} x^{\prime}=0$ where $x^{\prime}=\left(x_{1}, \cdots, x_{k}\right)$. By our inductive hypothesis (16) is true in $\mathscr{S}$ and hence $B^{\prime} x^{\prime}=0$. Then $B x=0$ since $x_{0}=0$. Thus $\mathfrak{X}$ is true in $\mathscr{S}$.

An easy consequence of Theorem 1 is

CoROLlaRY 1. A universal Horn sentence is true in $\left(\omega^{+}\right)^{*}$ if and only if it is true in every TFAG.

Proof. Suppose $\mathfrak{A}$ is a universal Horn sentence that is true in $\left(\omega^{+}\right)^{*}$. By elementary TFAG properties $\mathfrak{A}$ can be brought into the form (13) and as in Case 1 of the preceding proof (14) is true. Then as in the last paragraph of Case 1 we can show that $\mathfrak{U}$ is true in every TFAG. The converse is immediate.

Let $\omega^{\times}$be the TSG $(\omega-\{0\}, \cdot, 1) . \mathscr{S}$ is said to satisfy the Tarski condition $\times\left(\mathrm{TC}^{\times}\right)$if every system of equations in the variables $v_{0}, \cdots, v_{k}$ having a solution $x_{0}, \cdots, x_{k}$ in $\mathscr{S}$ with $x_{0} \neq 0$ also has a solution $x_{0}^{\prime}, \cdots, x_{k}^{\prime}$ in $\omega^{\times}$with $x_{0}^{\prime} \neq 1 . \mathscr{S}$ is said to have the Horn transfer property ${ }^{\times}\left(\mathrm{HTP}^{\times}\right)$if every universal Horn sentence which is true in $\omega^{\times}$is also true in $\mathscr{S}$. The following lemma is so well known that we find it difficult to find a source.

LEMMA 1. A Horn sentence is true in $\omega^{+}$if and only if it is true in $\omega^{\times}$.

Proof. Let $p_{0}, p_{1}, \ldots$ be the primes in increasing order and let $\Pi \omega^{+}$be the countable weak direct product of $\omega^{+}$. Consider the function $h$ which maps each $x \in \omega-\{0\}$ of the form $\Pi_{i<\omega} p_{i}^{a_{i}}$ into an infinite sequence consisting of the exponents of the $p_{i}$ 's in this prime 
power representation of $x$. Then $h$ is an isomorphism of $\omega^{\times}$onto $\Pi \omega^{+}$. Moreover every Horn sentence is true in $\Pi \omega^{+}$if and only if it is true in $\omega^{+}$.

CoROLlary 2. A TSG $\mathscr{S}$ has the $\mathrm{HTP}^{\times}$if and only if it satisfies the $\mathrm{TC}^{\times}$.

Proof. By Lemma $1 \mathscr{S}$ has the $\mathrm{HTP}^{\times}$if and only if it has the $\mathrm{HTP}^{+}$. At the beginning of the proof of Theorem 1 we showed that $\mathscr{S}$ satisfies the $\mathrm{TC}^{+}$if and only if every Horn sentence of the form (11) which is true in $\omega^{+}$is also true in $\mathscr{S}$. The same proof also gives this result for the $\mathrm{TC}^{\times}$(replacing $\omega^{+}$by $\omega^{\times}$). Thus by Lemma $1 \mathscr{S}$ satisfies the $\mathrm{TC}^{\times}$if and only if it satisfies the $\mathrm{TC}^{+}$.

COROLlaRY 3. A universal Horn sentence is true in $\left(\omega^{\times}\right)^{*}$ if and only if it is true in every TFAG.

Proof. $\left(\omega^{\times}\right)^{*}$ is isomorphic to $\Pi\left(\omega^{+}\right)^{*}$.

4. Isolic structures. Let $\Lambda^{+}=(\Lambda,+, 0), \Lambda^{\times}=(\Lambda-\{0\}, \cdot, 1)$, $\Delta^{+}=(\Delta,+, 0), \Delta^{\times}=(\Delta-\{0\}, \cdot, 1)$, and let ZF be set theory without the axiom of choice. Whenever we say that a sentence is true in $\Delta^{+}$ or $\Delta^{\times}$we shall mean that a properly relativized version of that sentence is a theorem of ZF (note that falseness is not the opposite of truth in this context).

As mentioned at the beginning of $\S 2, \Lambda^{+}$and $\Delta^{+}$are both TSGs. All of the properties needed to verify that $\Lambda^{\times}$is a TSG can be found in [2], and those needed to verify that $\Delta^{\times}$is a TSG can be found in [4]. Even before the general metatheorems of [8], Myhill had already shown that $\Lambda^{+}$and $\Lambda^{\times}$satisfied the $\mathrm{TC}^{+}$and $\mathrm{TC}^{\times}$respectively (unpublished). Subsequently we obtained the same results for $\Delta^{+}$and $\Delta^{\times}$. Shortly afterward we were informed by Tarski that he had long known of these results (for Dedekind cardinals), but had refrained from publishing them. Thus by Theorem 1 and Corollary 2 of the last section we know that $\Lambda^{+}$and $\Delta^{+}$have the $\mathrm{HTP}^{+}$and that $\Lambda^{\times}$and $\Delta^{\times}$have the $\mathrm{HTP}^{\times}$. Of course all this is redundant in the light of [8] and [4], but our argument has the attraction that the various TCs mentioned above are very easy to prove. We properly begin this section with an investigation of the first order theories of $\left(\Lambda^{\times}\right)^{*}$ and $\left(\Delta^{\times}\right)^{*}$.

In [7] Myhill gave a complete set of axioms for the theory of $\left(\Lambda^{+}\right)^{*}$. It is not hard to show that the theory of $\left(\Delta^{+}\right)^{*}$ is not complete and at the moment we do not even know if it is decidable. What is surprising is that $\left(\Delta^{\times}\right)^{*}$ has a complete decidable theory which in fact 
is the same as that of $\left(\Lambda^{\times}\right)^{*}$. [7] is based on methods devised by Smielew in [12] which we now expound. Let $\mathscr{G}=(G,+, 0)$ be a TFAG, $k, n \in \omega$, and $x_{0}, \cdots, x_{k} \in G . \quad x_{0}, \cdots, x_{k}$ are said to be strongly linearly independent $(\bmod n)$ if for each sequence of nonnegative integers $a_{0}, \cdots, a_{k}$, each $a_{i}<n$ and not all $=0$, there is no $y \in G$ such that $\sum_{i \leq k} a_{i} x_{i}=n y$. Let $\psi_{k n}$ be a sentence in our language $L$ saying that there exist $k$ elements strongly linearly independent $(\bmod n)$. The principal result of [12] when applied to TFAGs is

Proposition A. An extension of the theory of TFAGs is complete if and only if it is consistent and contains for any two integers $k>0, n>1$ either the sentence $\psi_{k n}$ or its negation.

Let MA be the set of sentences consisting of TFAG and $\left\{\psi_{k n} \mid k>0\right.$, $n>1\}$. In [7] it is shown that MA is a complete set of axioms for $\left(\Lambda^{+}\right)^{*}$. Here we show that the same holds for $\left(\Lambda^{\times}\right)^{*}$ and $\left(\Delta^{\times}\right)^{*}$.

THEOREM 2. (i) Every sentence of $\mathrm{MA}$ is true in $\left(\Lambda^{\times}\right)^{*}$. (ii) Every sentence of MA, when understood as referring to $\left(\Delta^{\times}\right)^{*}$, is a theorem of $\mathrm{ZF}$.

Proof. Since the proofs of (i) and (ii) are virtually the same we shall only prove (i). Let $p_{0}, p_{1}, \cdots$ be the primes in increasing order. We shall show that $p_{0}, \cdots, p_{k}$ are strongly linearly independent $(\bmod n)$ in $\left(\Lambda^{\times}\right)^{*}$ for any $n>1$. Let $a_{0}, \cdots, a_{k}$ be a sequence of nonnegative integers, each $a_{i}<n$ and not all $=0$. Then

$$
(\forall u, v)\left(v^{n}\left(\Pi_{i \leqq k} p_{i}^{a_{i}}\right)=u^{n} \longrightarrow u=0\right)
$$

is a universal Horn sentence in the language of [8] (although not in our language $L$; here we are discussing multiplication and exponentiation). By the following argument (17) is true in $\omega$. Let $x, y \in \omega$ and satisfy $y^{n}\left(\Pi_{i \leqq k} p_{i}^{a i}\right)=x^{n}$. If $x \neq 0$ then $y \neq 0$ so w.l.g. we may assume that $x$ and $y$ are relatively prime. If $p$ is any prime dividing $y$ then $p$ divides $x$. Thus $y=1$. If $p$ is any prime dividing $x$ then $p^{n}$ divides $\Pi_{i \leqq k} p_{i}^{a_{i}}$ contradicting the fact that each $a_{i}<n$. Thus $x=1$ giving $\Pi_{i \leqq k} p_{i}^{a_{i}}=1$ which is impossible since some $a_{i}$ is not 0 . Then (17) is true in $\Lambda$ by the metatheorems of [8] (of [4] in the cardinal case). Now if $p_{0}, \cdots p_{k}$ were not strongly linearly independent $(\bmod n)$ in $\left(\Lambda^{\times}\right)^{*}$ then there would exist $x, y \in \Lambda-\{0\}$ such that $y^{n}\left(\prod_{i \leqq k} p_{i}^{a}\right)=x^{n}$. But this contradicts the truth of (17) in $\Lambda$.

We summarize the situation as follows. $\left(\omega^{\times}\right)^{*},\left(\Lambda^{\times}\right)^{*},\left(\Lambda^{\times}\right)^{*}$, and $\left(\Lambda^{+}\right)^{*}$ all have the same complete theory $\mathrm{MA},\left(\omega^{+}\right)^{*}$ has a different complete theory, and there are propositions about $\left(\Delta^{+}\right)^{*}$ which are 
undecidable in ZF. Two remarks are in order. First, it is strange that $\left(\omega^{\times}\right)^{*}$ and $\left(\Lambda^{\times}\right)^{*}$ have the same theory. Usually when we extend structures from the finite to the Dedekind, about the only sentences which are preserved are the Horns. Second, it is strange that $\left(\Delta^{\times}\right)^{*}$ has a complete theory. Usually when we extend structures from the finite to the Dedekind, axiomatically in $\mathrm{ZF}$, we get a tremendous amount of incompleteness.

We leave TFAGs and return to TSGs. From $\S 3$ we know that $\omega^{+}, \omega^{\times}, \Lambda^{+}, \Lambda^{\times}, \Delta^{+}$, and $\Delta^{\times}$all satisfy the same universal Horn sentences, and that $\omega^{+}$and $\omega^{\times}$satisfy the same Horn sentences. Where do these similarities start breaking down? A fruitful start is from the cancellation law (8). Of course $\omega^{+}$and $\omega^{\times}$both satisfy (8); that $\Lambda^{+}$satisfies (8) is shown in [2], and that $\Delta^{+}$satisfies (8) is an elementary consequence of [14]. Note that when expressed in our language $L$, (8) has the form

$$
(\forall x, y, u)(\exists v)(n x+u=n y \longrightarrow x+v=y),
$$

a very simple $\mathrm{AE}$ Horn sentence. The following lemma is quite useful for an understanding of (8).

LEMMA 2. If $\mathscr{S}$ is a TSG then the following two conditions are equivalent. (i) (8) is true in $\mathscr{S}$. (ii) For every positive integer $n$ and $x \in S^{*}, n x \in S$ implies that $x \in S$.

Proof. Assume that (8) is true in $\mathscr{S}$, that $x, y \in S$, and that $n[x, y] \in S$. Then there is a $z \in S$ such that $[n x, n y]=n[x, y]=[z, 0]$ so that $n x=z+(n y), n y \leqq n x, y \leqq x$ by (8), and finally a $u \in S$ such that $y+u=x$. This implies $[x, y]=[u, 0] \in S$. Conversely assume (ii) and that $n x \leqq n y$. There is a $z \in S$ such that $(n x)+z=n y$. Then $n[y, x]=[n y, n x]=[z, 0] \in S$. By (ii) this implies that $[y, x] \in S$ and hence there is a $u \in S$ such that $[y, x]=[u, 0], y=x+u, x \leqq y$.

THEOREM 3. (i) (8) is not true in $\Lambda^{\times}$. (ii) When understood as referring to $\Delta^{\times},(8)$ is not a theorem of $\mathrm{ZF}$.

Proof. Since the proofs of (i) and (ii) are virtually the same we shall only prove (i). In view of Lemma 2 it will suffice to find a $d$ such that

$$
d \in\left(\Lambda^{\times}\right)^{*}-\Lambda^{\times} \text {and } d^{2} \in \Lambda^{\times} .
$$

For $x, y \in \Lambda$ write $y \mid x$ if $(\exists z \in \Lambda) x=y z$. Then (19) is equivalent to finding $a, b \in \Lambda-\{0\}$ such that

$$
\left(b^{2} \mid a^{2}\right) \text { but not }(b \mid a) \text {. }
$$


In [9] Nerode constructs $p, u$ satisfying

(P1) $p: \omega \rightarrow \omega$ is a strictly increasing recursive function, not eventually combinatorial, such that for every eventually combinatorial function $f$, not eventually linear, the composition $f \circ p$ is eventually combinatorial.

(P2) $u \in \Lambda-\omega$ and for any eventually recursive combinatorial functions $f$ and $g, f_{A}(u) \leqq g_{A}(u)$ implies that the difference function $g-f$ is eventually combinatorial.

Construct unary recursive combinatorial functions $p^{0}, p^{1}, q, g$, and $h$ such that for all $x \in \omega, p^{1}(x)-p^{0}(x)=p(x), h(x) p(x)=g(x)$, and $q(x)=[p(x)]^{2}$. Then in the language of [8], the following universal Horn sentences

$$
\begin{gathered}
(\forall x)\left([h(x)]^{2} q(x)=[g(x)]^{2}\right), \\
(\forall x, y)\left(h(x) y=g(x) \longrightarrow p^{0}(x)+y=p^{1}(x)\right)
\end{gathered}
$$

are true in $\omega$. By (P1) $q$ is eventually recursive combinatorial and hence by [8] (21), (22) are true in $\Lambda$. Let $a=g_{A}(u)$ and $b=h_{A}(u)$. Neither $g$ nor $h$ are bounded on $\omega$ because otherwise $p$ would be eventually combinatorial. Thus $a, b \in \Lambda-\{0\}$ since $u$ is infinite, and $b^{2} \mid a^{2}$ by (21). If $b \mid a$ then $h_{\Lambda}(u) y=g_{\Lambda}(u)$ for some $y \in \Lambda$. By (22) we get $p_{A}^{0}(u) \leqq p_{A}^{1}(u)$, so $p=p^{1}-p^{0}$ is eventually combinatorial by (P2), which contradicts (P1). Thus we have satisfied (20). In the cardinal case we use a set theoretic analogue of (P2) (cf. [5]).

This shows that although all of the specific TSGs introduced so far satisfy the same universal Horn sentences, they do not satisfy the same AE Horn sentences.

Let us now examine some of the properties of the structures $\Omega^{+}=(\Omega,+, 0), \Omega^{\times}=(\Omega-\{0\}, \cdot, 1), \Gamma^{+}=(\Gamma,+, 0)$, and $\Gamma^{\times}=(\Gamma-$ $\{0\}, \cdot, 1)$. First, because of general considerations, all of these structures satisfy (1) thru (4) and none satisfy (5). In [3] we showed that $\Omega^{\times}$does not satisfy (6). An easy way to see that $\Gamma^{\times}$does satisfy (6) is to argue that if it did, then taking $n=2$, we would obtain the cancellation law $x^{2}=y^{2} \rightarrow x=y$, which by a result of Tarski (cf. [13]) is equivalent to the axiom of choice. On the other hand $\Omega^{+}$and $\Gamma^{+}$satisfy (6) by [6] and [14] respectively. Recall that (5) was used to prove (7.3). Even though (5) is not available to us here, $\Gamma^{+}$satisfies (7.3) by the Cantor-Bernstein theorem (cf. [1]), and $\Omega^{+}$ satisfies (7.3) by Myhill's effective version of that theorem (cf. [2]). We argue that $\Omega^{\times}$and $\Gamma^{\times}$both satisfy (7.3). This amounts to showing that $x|y \wedge y| x \rightarrow x=y$ for $x, y \neq 0$. If $x a=y$ and $y b=x$ then $(x a) b=x$. Thus $x \leqq x a \leqq(x a) b \leqq x$ and hence $x=x a=y$ by the Cantor-Bernstein theorem. Thus the canonical $\leqq$ is a partial order 
on all of our structures. However none of them satisfy (7.6), for then (5) would follow. The same argument shows that neither $\Omega^{\times}$ nor $\Gamma^{\times}$satisfies (8) for then (6) would follow. On the other hand the fact that $\Gamma^{+}$satisfies (6) is really a consequence of the fact that it satisfies (8) (cf. [14]). We round out the situation with the following theorem which was first obtained by $\mathrm{A}$. Nerode and later by the author.

\section{THEOREM $4 . \Omega^{+}$satisfies (8).}

Proof. First we introduce some notation. We use lower case Greek letters for subsets of $\omega . j$ is the usual pairing function with first, second inverses $k, l$ respectively. Let $\alpha \times \beta=\{j(x, y) \mid x \in \alpha \wedge$ $y \in \beta\}$ and $\nu(n)=\{x \in \omega \mid x<n\}$. If $f$ is a function let $\delta f, \rho f$ denote the domain, range of $f$ respectively.

We start our proof by assuming that $n$ is a positive integer, $A_{0}$ and $A_{1}$ are in $\Omega$, and $n A_{0} \leqq n A_{1}$. Let $\alpha_{i} \in A_{i}$ and $\bar{\alpha}_{i}=\alpha_{i} \times \nu(n)$. Then $\bar{\alpha}_{i} \in n A_{i}$ and there a one-one partial recursive function $p$ and disjoint recursively enumerable sets, $\sigma$ and $\tau$, such that $\bar{\alpha}_{0} \leqq \delta p$, $p\left(\bar{\alpha}_{0}\right) \subseteq \bar{\alpha}_{1}, p\left(\bar{\alpha}_{0}\right) \subseteq \tau$, and $\bar{\alpha}_{1}-p\left(\bar{\alpha}_{0}\right) \subseteq \sigma$. We make the following simplifying assumptions. (i) $\alpha_{0}$ is separable from $\alpha_{1}$ (cf. [2]), in fact we may assume that every $x \in \alpha_{0}$ is even and every $y \in \alpha_{1}$ is odd. (ii) If $x \in \delta p$ then $k(x)$ is even and $l(x)<n$, if $y \in \rho p$ then $k(y)$ is odd and $l(y)<n$. (iii) $\rho p=\tau$ and every element in $\sigma \cup \tau$ is odd. Let $f, g$ be recursive functions enumerating $\sigma, \tau$ respectively and let $\sigma^{s}=$ $\{f(x) \mid x \leqq s\}$ and $\tau^{s}=\{g(x) \mid x \leqq s\}$. Let $h$ be a recursive function such that the graph of $p$ is $\{(k h(x), l h(x)) \mid x \in \omega\}$ and let $p^{s}$ be the finite function whose graph is $\{(k h(x), \operatorname{lh}(x)) \mid x \leqq s\}$. We let $\beta_{0}^{s}=$ $\left\{k(x) \mid x \in \delta p^{s}\right\}, \beta_{1}^{s}=\left\{k(y) \mid y \in \rho p^{s}\right\}$ and $\beta^{s}=\beta_{0}^{s} \cup \beta_{1}^{s}$. Define a predicate $\Phi^{s}(x, y) \equiv_{D F} p^{s}(x)=y \vee p^{s}(y)=x \vee k(x)=k(y)$. If $x, y \in \beta^{s}$ we say that $x$ is linked to $y$ by stage $s$ (in symbols $x \sim{ }^{s} y$ ) if there is a finite sequence $\left\{u_{0}, \cdots, u_{m}\right\} \cong \delta p^{s} \cup \rho p^{s}$ such that $k u_{0}=x, k u_{m}=y$, and $\Phi^{s}\left(u_{i}, u_{i+1}\right)$ for each $i<m$. We say that $x \in \beta_{0}^{s}$ is released by stage $s$ if $\{x\} \times \nu(n) \subseteq \delta p^{s}$ and $y \in \beta_{1}^{s}$ is releasee by stage $s$ if $\{y\} \times \nu(n) \subseteq$ $\sigma^{s} \cup \tau^{s}$ and $(\{y\} \times \nu(n))-\rho p^{s} \subseteq \sigma^{s}$.

We define finite functions $q^{s}$ as follows. $q^{0}$ is nowhere defined. Assume $q^{s}$ is defined. Find the least $x \in \beta_{0}^{s}-\delta q^{s}$ and the least $y \in \beta_{1}^{s}-\rho q^{s}$ such that $x$ is linked to $y$ by stage $s$, and $x, y$ are both released by stage $s$. If there is no such pair let $q^{s+1}=q^{s}$. If there is such a pair let $q^{s+1}$ be the same as $q^{s}$ on $\delta q^{s}$ and let $q^{s+1}(x)=y$. We then define $q(x)=y \equiv_{D F}(\exists s) q^{s}(x)=y$. Clearly $q$ is a one-one function, and since we can effectively tell at any stage whether objects are linked or released, $q$ is a partial recursive function.

In order to prove that $A_{0} \leqq A_{1}$ it suffices to show that 


$$
\begin{gathered}
\alpha_{0} \cong \delta q, \text { and } q\left(\alpha_{0}\right) \cong \alpha_{1} \text { and } \\
q\left(\alpha_{0}\right) \text { is separable from } \alpha_{1}-q\left(\alpha_{0}\right) .
\end{gathered}
$$

Several definitions and lemmas are needed. Notice that $\sim^{s}$ is an equivalence relation on $\beta^{s}$.

Lemma 3. If $x, y \in \beta^{s}$ and $x \sim{ }^{s} y$ then $x \in \alpha_{0} \cup \alpha_{1}$ if and only if $y \in \alpha_{0} \cup \alpha_{1}$.

Proof. By induction on the length of the chain $u_{0}, \cdots, u_{m}$ connecting $x$ and $y$. It will suffice to show that if $u_{0}=j\left(x, m_{0}\right), u_{1}=$ $j\left(y, m_{1}\right),\left\{u_{0}, u_{1}\right\} \subseteq \delta p^{s} \cup \rho p^{s}$, and $\Phi^{s}\left(u_{0}, u_{1}\right)$ then $x \in \alpha_{0} \cup \alpha_{1}$ if and only if $y \in \alpha_{0} \cup \alpha_{1}$. Only two cases are of any importance, the rest either trivial or following by symmetry.

Case 1. Assume $x \in \alpha_{0}$ and $p\left(u_{0}\right)=u_{1}$. Then $u_{0} \in \bar{\alpha}_{0}, u_{1} \in \bar{\alpha}_{1}$ and hence $y \in \alpha_{1}$.

Case 2. Assume $y \in \alpha_{1}$ and $p\left(u_{0}\right)=u_{1}$. Then $u_{1} \in \bar{\alpha}_{1} \cap \rho p$. But $\bar{\alpha}_{1} \cap \rho p=\bar{\alpha}_{1} \cap p\left(\bar{\alpha}_{0}\right)$ by our simplifying assumptions. Hence $u_{0} \in \bar{\alpha}_{0}$ and $x \in \alpha_{0}$.

For $x \in \beta^{s}$ : let $\gamma^{s}(x)=\left\{y \in \beta^{s} \mid x \sim^{s} y\right\}, \gamma_{0}^{s}(x)=$ the even elements in $\gamma^{s}(x)$, and $\gamma_{1}^{s}(x)=$ the odd elements in $\gamma^{s}(x)$. By Lemma 3 each $\gamma^{s}(x)$ is either contained in $\alpha_{0} \cup \alpha_{1}$ or disjoint from it. Say $x$ is linked to $y$ (in symbols $x \sim y$ ) if $(\exists s) x \sim^{s} y$. Clearly $\sim$ is an equivalence relation. Let $\gamma(x)=\{y \in \omega \mid x \sim y\}, \gamma_{0}(x)=$ the even elements in $\gamma(x)$, and $\gamma_{1}(x)=$ the odd elements in $\gamma(x)$. From the corresponding property of $\gamma^{s}(x)$ we find that each $\gamma(x)$ is either contained in $\alpha_{0} \cup \alpha_{1}$ or disjoint from it. The heart of our proof (and the reason why we required that if $q^{s}(x)=y$ then $x, y$ were released at some stage prior to $s$ ) is given in the next lemma.

LemMA 4. If $x \in \alpha_{0}$ then $x \in \delta q$.

Proof. Assume that $x \in \alpha_{0}$. Then $p$ maps $\gamma_{0}(x) \times \nu(n)$ one-one into $\gamma_{1}(x) \times \nu(n)$. For any set $\delta$ let $|\delta|=$ the cardinality of $\delta$. Then $\left|\gamma_{0}(x)\right| \leqq\left|\gamma_{1}(x)\right|$ by ordinary cancellation. If $q\left(x^{\prime}\right) \in \gamma_{1}(x)$ then $x^{\prime} \in \gamma_{0}(x)$ since the first and second element of any pair in the graph of $q$ must be linked. If $\gamma_{0}(x)$ is finite there is a stage $s$ such that every $x^{\prime} \in \gamma_{0}(x)$ is released by stage $s$, and at least $\left|\gamma_{0}(x)\right|$ elements of $\gamma_{1}(x)$ are released by stage $s$. Clearly $q(x)$ is defined. The interesting case is when $\gamma_{0}(x)$ is infinite. For then unless special care is taken any candidate $y$ for $q(x)$ might already be in $\rho q$ prior to being linked 
with $x$. Assuming that $\gamma_{0}(x)$ is infinite we can find a stage $s$ such that $x$ is released by stage $s$ and for all $x^{\prime}<x, x^{\prime} \in \delta q \equiv x^{\prime} \in \delta q^{s}$. Now $\gamma_{1}(x)$ is infinite so if $x$ is not already in $\delta q^{s}$ there will be a least stage $t \geqq s$ such that $x \notin \delta q^{t}$ and a least $y \in \gamma_{1}^{t}(x)$ such that $y$ is released by stage $t$, but by no stage $t^{\prime}<t$. This insures that $y \notin \rho q^{t}$. Then $q^{t+1}(x)=y$ since no $x^{\prime}<x$ can be put into $\delta q$ after stage $s$.

By interchanging the roles of range and domain in the preceding proof we easily obtain

Lemma 5. If $y \in \alpha_{1}$ and $\gamma_{0}(y)$ is infinite then $y \in \rho q$.

Now assume that $x \in \delta q$. Then by our construction $q(x) \in \gamma_{1}(x)$. It follows from Lemma 3 that $x \in \alpha_{0}$ if and only if $q(x) \in \alpha_{1}$. By Lemma $4, \alpha_{0} \subseteq \delta q$ and hence $q$ maps $\alpha_{0}$ into $\alpha_{1}$ proving (23). Moreover, $q\left(\alpha_{0}\right)=\alpha_{1} \cap \rho q$ so that (24) will follow if we can find a recursively enumerable set $\theta$ which is disjoint from $\rho q$ and contains $\alpha_{1}-\rho q$. Put $y$ in $\theta$ if either

$$
\{y\} \times \nu(n) \subseteq \sigma
$$

or there is an $s$ satisfying (26) and (27) below.

$$
y \in \beta_{1}^{s} \text { and every element in } \gamma^{s}(y) \text { is released by stage } s \text {. }
$$

$$
\gamma_{0}^{s}(y) \subseteq \delta q^{s} \text { and } y \notin \rho q^{s} \text {. }
$$

Clearly $\theta$ is recursively enumerable. Suppose that $y \in \alpha_{1}-\rho q$. If $y \notin \beta_{1}^{s}$ for every $s$ then (25) is true. Otherwise $\gamma(y)$ is defined. If $\gamma_{0}(y)$ is infinite then $y \in \rho q$ by Lemma 5 . Hence $\gamma_{0}(y)$ is finite. But then $\gamma(y)$ is finite and $\gamma(y)=\gamma^{s}(y)$ for sufficiently large $s$. Every element in $\gamma^{s}(y)$ is eventually released and $\gamma_{0}^{s}(y) \subseteq \alpha_{0} \leqq \delta q$. Hence we can satisfy (26) and (27) showing that $y \in \theta$, i.e., $\alpha_{1}-\rho q \subseteq \theta$. Now suppose that $y \in \theta$. If (25) holds then $y$ certainly is not in $\rho q$. If (26) holds then $\gamma(y)=\gamma^{s}(y)$ and if (27) also holds then $y \notin \rho q$ because if $q(x)=y$ for some $x$ then $x \in \gamma_{0}^{s}(y)$ and $y \in \rho q^{s}$ contradicting (27). Thus we have shown that $\theta$ is disjoint from $\rho q$. This completes the proof of Theorem 4 .

The notion of "released" used in the proof of this theorem is due to Friedberg (cf. [6]). The anthropomorphic way in which we have used this term (and "linked" as well) is due to Nerode (cf. [10]). We have already worked out the universal theory of linear inequalities in $\Omega^{+}$(in the style of 10]) and intend to present it in a future publication. 


\section{REFERENCES}

1. F. Bernstein, Untersuchungen aus der Mengelehre, Math. Annalen, 61 (1905), 117155.

2. J. C. E. Dekker and J. Myhill, Recursive Equivalence Types, Univ. of Calif. Publs. in Math., (NS) 3, \#3 (1960), 67-214.

3. E. Ellentuck, Solution to a problem of R. Friedberg, Math. Zeit., 82 (1963), 101103.

4. - A choice free theory of Dedekind cardinals, J. Symbolic Logic, 34 (1969), $70-84$.

5. - Extension methods in cardinal arithmetic, Trans. Amer. Math. Soc., 149 (1970), 307-325.

6. R. Friedberg, The uniqueness of finite division for recursive equivalence types, Math. Zeit., 75 (1961), 3-7.

7. J. Myhill, Elementary properties of the group of isolic integers, Math. Zeit., 78 (1962), 126-130.

8. A. Nerode, Extensions to isols, Ann. of Math., 73 (1961), 362-403.

9. - Non-linear combinatorial functions of isols, Math. Zeit., 86 (1965), 410-424.

10. - Additive relations among recursive equivalence types, Math. Annalen, 159 (1965), 329-343.

11. W. Sierpiński, Sur l'égalité $2 m=2 n$ pour les nombres cardinaux, Fund. Math., 3 (1922), 1-6.

12. W. Szmielew, Elementary properties of Abelian groups, Fund. Math., 41 (1955), 203-271.

13. A. Tarski, Sur quelques théorèmes qui équivalent à l'axiome de choix, Fund. Math., 5 (1924), 147-154.

14. - Cancellation laws in the arithmetic of cardinals, Fund. Math., 36 (1949), $77-92$.

Received August 27, 1974. Partially supported by NSF GP-28348.

Rutgers, The State University 



\section{PACIFIC JOURNAL OF MATHEMATICS}

\section{EDITORS}

RICHARD ARENS (Managing Editor) University of California

Los Angeles, California 90024

\section{R. A. BEAUMONT}

University of Washington Seattle, Washington 98105
J. DugundjI Department of Mathematics University of Southern Californı Los Angeles, California 90007

D. Gilbarg AND J. Milgram Stanford University Stanford, California 94305

\section{ASSOCIATE EDITORS}

E. F. BECKENBACH

B. H. NeUMANN

F. WOLF

K. YosHidA

\section{SUPPORTING INSTITUTIONS}

UNIVERSITY OF BRITISH COLUMBIA CALIFORNIA INSTITUTE OF TECHNOLOGY UNIVERSITY OF CALIFORNIA MONTANA STATE UNIVERSITY UNIVERSITY OF NEVADA NEW MEXICO STATE UNIVERSITY OREGON STATE UNIVERSITY UNIVERSITY OF OREGON OSAKA UNIVERSITY

\author{
UNIVERSITY OF SOUTHERN CALIFORNIA \\ STANFORD UNIVERSITY \\ UNIVERSITY OF TOKYO \\ UNIVERSITY OF UTAH \\ WASHINGTON STATE UNIVERSITY \\ UNIVERSITY OF WASHINGTON \\ AMERICAN MATHEMATICAL SOCIETY \\ NAVAL WEAPONS CENTER
}




\section{Pacific Journal of Mathematics}

\section{Vol. 61, No. $1 \quad$ November, 1975}

Jiří Adámek, V. Koubek and Věra Trnková, Sums of Boolean spaces represent every

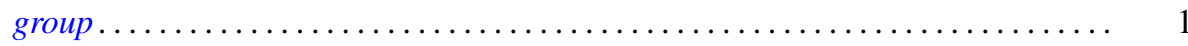

Richard Neal Ball, Full convex l-subgroups and the existence of $a^{*}$-closures of

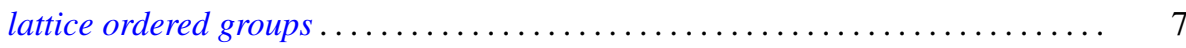

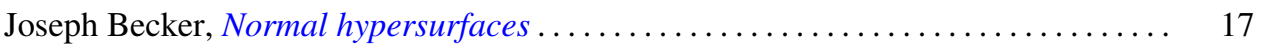

Gerald A. Beer, Starshaped sets and the Hausdorff metric . . . . . . . . . . . . . 21

Dennis Dale Berkey and Alan Cecil Lazer, Linear differential systems with

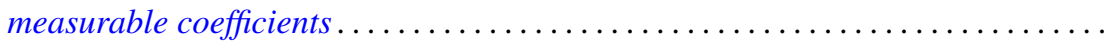

Harald Boehme, Glättungen von Abbildungen 3-dimensionaler

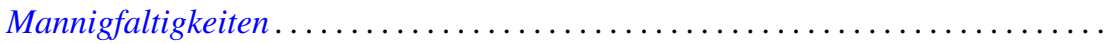

Stephen LaVern Campbell, Linear operators for which $T^{*} T$ and $T+T^{*}$

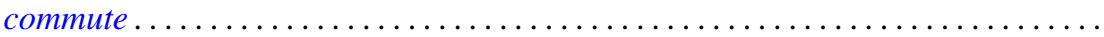

H. P. Dikshit and Arun Kumar, Absolute summability of Fourier series with



Andrew George Earnest and John Sollion Hsia, Spinor norms of local integral

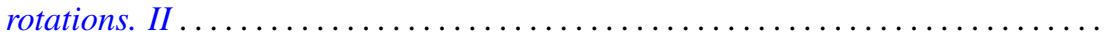

Erik Maurice Ellentuck, Semigroups, Horn sentences and isolic structures .........

Ingrid Fotino, Generalized convolution ring of arithmetic functions . . . . . . . . . . .

Michael Randy Gabel, Lower bounds on the stable range of polynomial rings .......

Fergus John Gaines, Kato-Taussky-Wielandt commutator relations and

characteristic curves

Theodore William Gamelin, The polynomial hulls of certain subsets of $C^{2}$

R. J. Gazik and Darrell Conley Kent, Coarse uniform convergence spaces. . .

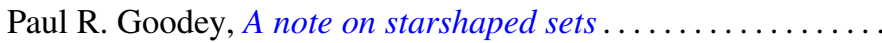

Eloise A. Hamann, On power-invariance

M. Jayachandran and M. Rajagopalan, Scattered compactification for $N \cup\{P\}$. . .

V. Karunakaran, Certain classes of regular univalent functions .

John Cronan Kieffer, A ratio limit theorem for a strongly subadditive set function in a locally compact amenable group .................

Siu Kwong Lo and Harald G. Niederreiter, Banach-Buck measure, density, and uniform distribution in rings of algebraic integers ........

Harold W. Martin, Contractibility of topological spaces onto metric spaces ....

Harold W. Martin, Local connectedness in developable spaces .

A. Meir and John W. Moon, Relations between packing and covering numbers of a tree.



Donald J. Newman and I. J. Schoenberg, Splines and the logarithmic function . . . . 241

M. Ann Piech, Locality of the number of particles operator....

Fred Richman, The constructive theory of $K T$-modules .......

Gerard Sierksma, Carathéodory and Helly-numbers of

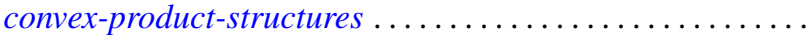

Raymond Earl Smithson, Subcontinuity for multifunctions .... . . 